
(

\title{
The potential of low molecular weight heparin to mitigate cytokine storm in severe COVID-19 patients: a retrospective clinical study
}

Chen Shi\#, Cong Wang\#, Hanxiang Wang\#, Chao Yang, Fei Cai, Fang Zeng, Fang Cheng, Yihui Liu, Taotao Zhou, Bin Deng, Israel Vlodavsky, Jin-Ping Li, Yu Zhang*

\#Contributed equally

*Corresponding author

Department of Pharmacy, Union Hospital, Tongji Medical College, Huazhong University of Science and Technology, Wuhan, China; Hubei Province Clinical Research Center for Precision Medicine for Critical Illness, Wuhan, China (C Shi PhD, Y Zhang PhD); Department of Pharmacy, Union Hospital, Tongji Medical College, Huazhong University of Science and Technology, Wuhan, China (C Wang MD, H Wang MD, F Zeng MD, F Cheng MD, Y Liu PhD, T Zhou MD, B Deng PhD); Department of Medical Biochemistry and Microbiology, Uppsala University, Uppsala, Sweden (JP Li, PhD); Technion Integrated Cancer Center, Rappaport Faculty of Medicine, Technion, Israel (I Vlodavsky, PhD); Department of Vascular Surgery, Union Hospital, Tongji Medical College, Huazhong University of Science and Technology, Wuhan, China (C Yang MD, F Cai MD)

Correspondence to:

Prof Yu Zhang PhD, Department of Pharmacy, Union Hospital, Tongji Medical College, Huazhong University of Science and Technology, Wuhan, 430000, China, whxhzy@163.com 40 41 2

NOTE: This preprint reports new research that has not been certified by peer review and should not be used to guide clinical practice. 


\section{Summary:}

Background On March 11, 2020, the World Health Organization declared its assessment of COVID-19 as a global pandemic. However, specific antiviral drugs are still unavailable, and pateints are managed by multiple complementary treatments.

Methods The electronic medical records of COVID-19 patients where basic information, complete blood count, coagulation profile, inflammatory cytokines and serum biochemical indicators in 42 patients with COVID-19 (21 of whom were treated with low molecular weight heparin (LMWH), and 21 without LMWH) that were retrospectively analyzed to compare and evaluate the effect of LMWH treatment on disease progression.

Findings 42 patients with COVID-19 treated at the hospital between February 1 and March 15, 2020, were selected for the study, of which 21 underwent LMWH treatment (LMWH group), and 21 did not (Control), during hospitalization. Changes in the percentage of lymphocytes in the LMWH group before and after LMWH treatment were significantly different from those in the control group $(11 \cdot 10 \pm 9 \cdot 50$ vs. $3 \cdot 08 \pm 9 \cdot 66, p=0 \cdot 011$, respectively). Changes in the levels of D-dimer and fibrinogen degradation products (FDP) in the LMWH group before and after LMWH treatment were significantly different from those in the control group $(-2 \cdot 85 \pm 3 \cdot 90,-0 \cdot 05 \pm 0.85, p=0 \cdot 002 ;-9 \cdot 05 \pm 13 \cdot 14,-1 \cdot 78 \pm 3 \cdot 15$, $p=0 \cdot 035)$. Strikingly, in the LMWH group, IL-6 levels were significantly reduced after LMWH treatment $(47 \cdot 47 \pm 58 \cdot 86,15 \cdot 76 \pm 25 \cdot 71, p=0 \cdot 006)$. Besides, the changes in IL-6 levels in the LMWH group before and after LMWH treatment were significantly different from those in the control group $(-32 \cdot 46 \pm 65 \cdot 97,14 \cdot 96 \pm 151 \cdot 09, p=0 \cdot 031)$.

Interpretation LMWH improves the coagulation dysfunction of COVID-19 patients and exerts anti-inflammatory effects by reducing IL-6 and increasing lymphocyte \%. It appears that LMWH can be used as a potential therapeutic drug for the treatment of COVID-19, paving the way for a subsequent well-controlled clinical trial.

Funding National Natural Science Foundation of China (No. 81603037 to SC) and the National Key Research and Development Plan of China(2017YFC0909900).

Keywords LMWH; COVID-19; Lymphocytes\%; IL-6; Cytokine Storm.

\section{Introduction}

On March 11, 2020, the World Health Organization (WHO) declared its assessment of COVID-19 as a global pandemic. SARS-CoV-2 is characterized by a long incubation period, high infectivity, and multiple routes of transmission., ${ }^{1,2}$ According to real-time WHO statistics, the total number of confirmed cases of COVID-19 worldwide as of April 6, 2020 has exceeded a million with more than 70000 deaths. However, no effective medicines are currently available, so patients are treated symptomatically. Given the rapid spread of COVID-19 and the high mortality rate in severe cases, there is an urgent need to promptly control the occurence of a severe disease. A better understanding of the mechanisms of pathological changes will help to screen reliable drugs out of presently existing medications.

Lymphopenia and inflammatory cytokine storm are typical abnormalities observed in highly pathogenic coronavirus infections (such as SARS and MERS), ${ }^{3}$ believed to be associated with disease severity. ${ }^{4-6}$ Several clinical studies revealed that cytokine storms are important mechanisms underlying disease exacerbation and death of COVID-19 patients. ${ }^{4-6}$ Particularly, IL-6 levels in severely ill patients were significantly higher than in mild cases. ${ }^{7}$ IL- 6 is one of the core cytokines that are consistently 
found to be elevated in the plasma of patients with cytokine storm, ${ }^{8}$ contributing to many of the key symptoms of cytokine storm, such as vascular leakage, activation of the complement and coagulation cascades, inducing disseminated intravascular coagulation (DIC). ${ }^{9,10}$ Reducing the level and activity of IL-6 may contribute to prevent or even reverse the cytokine storm syndrome, ${ }^{11}$ thereby improving the condition of patients with COVID-19.

Substantial studies have reported that low molecular weight heparin (LMWH) has various non-anticoagulant properties ${ }^{12}$ that play an anti-inflammatory role by reducing the release of IL-6. ${ }^{13-15}$ However, the anti-inflammatory effects of LMWH in COVID-19 are currently unknown. By analyzing the relieving effect of LMWH in patients with COVID-19 our retrospective cohort study demonstrates, for the first time, the significant benifiical effect of LMWH in controlling ctytokine storm. This approach is believed to delay disease progression in COVID-19 patients, strongly encuraging a well-controlled clinical practice (Fig. 1).

\section{Methods}

\section{Research subjects}

To investigate the therapeutic effect of LMWH on COVID-19, we conducted a retrospective study. All cases in this study were located at Union Hospital, Tongji Medical College, Huazhong University of Science and Technology (Wuhan, Hubei Province, China), a designated treatment hospital for patients with COVID-19. This study was approved by the institutional review board of the hospital. In total, we retrospectively collected the electronic medical records of 42 patients with COVID-19, the admission data for these patients was from February 1, 2020, to March 15, 2020 (Fig. 2 shows the case inclusion flowchart), of which 21 underwent LMWH treatment (defined as LMWH group), and 21 did not (defined as Control group ) ( Table 1), during hospitalization.

Inclusion criteria: (1) met the diagnostic standards of novel coronavirus pneumonia (7th edition) formulated by the National Health Commission of China; (2) experienced any of the following: shortness of breath, respiration $\operatorname{rate}(\mathrm{RR}) \geq 30$ breaths/minute; resting oxygen saturation $\leq 93 \%$; $\mathrm{PaO} 2 / \mathrm{FiO} 2 \leq 300 \mathrm{mmHg}$; lung imaging showing significant lesion progression of $>50 \%$ within $24-48$ h, and a severe clinical classification; (3) age $\geq 18$ years; (4) no previous history of bronchiectasis, bronchial asthma, or other respiratory diseases; (5) no immunosuppressant or glucocorticoid use during treatment.

Exclusion criteria: (1) patients with severe systemic diseases and other acute or chronic infectious diseases; (2) patients with liver and kidney insufficiency or congenital heart disease; (3) patients who had been treated with LMWH in the previous three months; (4) patients with a prior history of mental illness; (5) pregnant or lactating women; (6) patients clinically classified as critically ill or housed in the intensive care unit (ICU); (7) patients allergic to LMWH or contraindicated for LMWH.

\section{Data collection}

The basic information, complete blood count, coagulation profile, inflammatory cytokines, and serum biochemical indicators (including liver function, kidney function, lactate dehydrogenase, C-reactive protein (CRP) and electrolytes) of 42 patients with COVID-19 were retrospectively analyzed. Two researchers also independently reviewed the data collection forms to double-check the data collected.

\section{Statistical analysis}

Data analysis was performed using SPSS 22.0 statistical software. Data are expressed as mean \pm standard deviation (SD). GraphPad 6.0 software was used for plotting. Differences between groups 
were evaluated using the T-test for measurement data, the Chi-square test for count data, and the Kruskal-Wallis nonparametric test between groups (independent samples) and within groups (related samples). Differences of $p<0 \cdot 05$ were considered statistically significant.

\section{Results}

\section{General characteristics of patients with COVID-19}

As shown in Table 2, the LMWH group consisted of 13 males and eight females aged between 42 and 91 years (median age $=69 \cdot 0$ years), and the Control group consisted of 14 males and seven females aged between 40 and 84 years (median age $=69 \cdot 0$ years). There were no significant differences in comorbidities, such as hypertension, diabetes, cardiovascular disease, and cancer, between the two groups. Similarly, there were no significant differences in coronavirus pneumonia onset symptoms, including fever (body temperature $\geq 37 \cdot 3^{\circ} \mathrm{C}$ ), cough, sputum, chest distress or asthma, myalgia, fatigue, anorexia, diarrhea, and nausea and vomiting. Similarly, there was no significant difference in antiviral treatment between the two groups. These results indicate that the general characteristics of the two groups of patients were consistent and comparable.

LMWH has no effect on the duration of conversion to negative and the length of patient hospitalization

As shown in Table 2, the number of days to convert virus to negative (time from admission to virus shedding) was $20 \cdot 0$ days (IQR $11 \cdot 0-31 \cdot 0$ ) in the LMWH group and $19 \cdot 0$ days (IQR $12 \cdot 0-30 \cdot 0$ ) in the Control group $(p=0 \cdot 46)$; the difference between the two groups was not significant. Similarly, the length of hospital stay was $29 \cdot 0$ days (IQR $17 \cdot 0-42 \cdot 0$ ) in the LMWH group and $27 \cdot 0$ days (IQR 24.0-31.0) in the Control group ( $p=0 \cdot 41)$; the difference between the two groups was not significant. Notably, all patients in the LMWH group and the Control group showed overall improvement after treatment.

\section{Effect of LMWH on blood routine characteristics}

As shown in Fig.3A-D, there was no significant difference in red blood cells (RBC), white blood cells (WBC), monocyte\%, and neutrophil\% levels between the two groups. Fig. 3E reveals no significant difference in lymphocyte\% between the LMWH and Control groups before LMWH treatment $(18 \cdot 84 \pm 8 \cdot 24,22 \cdot 42 \pm 8 \cdot 74, p=0 \cdot 144)$. There was also no significant difference in lymphocyte $\%$ between the LMWH and Control groups after LMWH treatment $(29 \cdot 94 \pm 7 \cdot 92,25 \cdot 65 \pm 10 \cdot 10, p=0 \cdot 215)$. However, patients in the LMWH group had a significantly increased percentage of lymphocytes after LMWH treatment $(18 \cdot 84 \pm 8 \cdot 24,29 \cdot 94 \pm 7 \cdot 92, p=0 \cdot 00048)$. Besides, the changes in lymphocyte $\%$ in patients of the LMWH group before and after LMWH treatment were significantly different from those in the Control group $(11 \cdot 10 \pm 9 \cdot 50,3 \cdot 08 \pm 9 \cdot 66, p=0 \cdot 011)$.

\section{Effect of LMWH on coagulation parameters}

There was no significant difference in thrombin time (TT, Fig. 3F), activated partial thromboplastin time (APTT, Fig. 3G), and prothrombin time (PT, Fig. 3H) levels between the two groups. As shown in Fig. 3I, the levels of D-dimer in the LMWH group were significantly higher compared to those in the Control group before treatment $(3 \cdot 75 \pm 4 \cdot 04,1 \cdot 23 \pm 1 \cdot 15, p=0 \cdot 009)$. There was no significant difference in D-dimer levels between the LMWH and Control groups after LMWH treatment $(0 \cdot 90 \pm 0 \cdot 44$, $1 \cdot 00 \pm 1 \cdot 06, p=0 \cdot 368)$. Upon LMWH treatment, the D-dimer levels were significantly reduced in the LMWH group $(3 \cdot 75 \pm 4 \cdot 04,0 \cdot 90 \pm 0 \cdot 44, p=0 \cdot 001)$ (Fig. 3I). The changes in D-dimer levels in patients in 
the LMWH group before and after LMWH treatment were significantly different from those in the Control group $(-2 \cdot 85 \pm 3 \cdot 90,-0 \cdot 05 \pm 0 \cdot 85, p=0 \cdot 002)$. As shown in Fig. 3J, the levels of fibrinogen degradation products (FDP) in the LMWH group were significantly higher compared to those in the Control group before treatment $(14 \cdot 35 \pm 14 \cdot 6,4 \cdot 05 \pm 3 \cdot 9, p=0 \cdot 002)$. There was no significant difference in FDP levels between the LMWH and Control groups after LMWH treatment $(2 \cdot 64 \pm 1 \cdot 16,3 \cdot 59 \pm 4 \cdot 00$, $p=0 \cdot 959$ ). In the LMWH group, FDP levels were significantly reduced after LMWH treatment $(14 \cdot 35 \pm 14 \cdot 6,2 \cdot 64 \pm 1 \cdot 16, p=0 \cdot 001)$. The changes in FDP levels in patients of the LMWH group before and after LMWH treatment were significantly different from those in the Control group $(-9 \cdot 05 \pm 13 \cdot 14$, $-1 \cdot 78 \pm 3 \cdot 15, p=0 \cdot 035)$. However, there was no significant difference in fibrinogen (FIB, Fig. 3K), antithrombin (ATIII, Fig. 3L) and international normalized ratio (INR, Fig. 3M) levels between the two groups.

\section{Effect of LMWH on CRP levels}

As shown in Fig. 3N, LMWH treatment had no significant effect on CRP levels. There is no difference between the two groups of patients before LMWH treatment $(31 \cdot 15 \pm 26 \cdot 62,29 \cdot 00 \pm 23 \cdot 79, p=0 \cdot 497)$, nor after LMWH treatment $(8 \cdot 95 \pm 10 \cdot 44,8 \cdot 76 \pm 16 \cdot 66, p=0 \cdot 620)$. Consequently, there were no significant differences in the changes in CRP levels between the two groups of patients before and after LMWH treatment $(-22 \cdot 62 \pm 23 \cdot 79,-20 \cdot 23 \pm 33 \cdot 91, p=0 \cdot 660)$.

\section{Effect of LMWH on cytokine levels in patients with COVID-19}

Finally, we have analyzed the levels of inflammatory cytokines in the two groups. As shown in Fig. 4, there were no significant differences in the levels of IL-2, IL-4, IL-10, TNF- $\alpha$, and IFN- $\gamma$ between the LMWH treated and untreated groups. Notably, application of LMWH significanlty lowered the level of IL-6. As shown in Fig. 4B, both groups had a high level of IL-6, and there was no significant difference between the LMWH and Control groups before treatment $(47 \cdot 47 \pm 58 \cdot 86,63 \cdot 27 \pm 96 \cdot 27, p=0 \cdot 950)$. In contrast, after LMWH treatment, the levels of IL-6 in the LMWH group were significantly lower compared to those in the Control group $(15 \cdot 76 \pm 25 \cdot 71,78 \cdot 24 \pm 142 \cdot 41, p=0 \cdot 00039)$. Accordingly, IL-6 levels in the LMWH group were significantly reduced after LMWH treatment $(47 \cdot 47 \pm 58 \cdot 86$, $15 \cdot 76 \pm 25 \cdot 71, p=0 \cdot 006$ ). Similarly, the changes in IL-6 levels in the LMWH group before and after

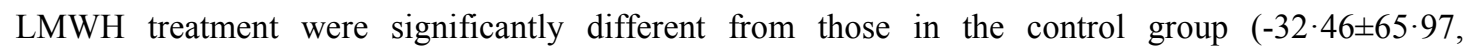
$14 \cdot 96 \pm 151 \cdot 09, p=0 \cdot 031)$.

\section{Discussion}

Cytokine storms are associated with deterioration in several infectious diseases, including SARS and avian influenza, ${ }^{3,16}$ and are an important cause for exacerbation in patients. ${ }^{17}$ In recent years, studies have revealed that heparin has various non-anticoagulant properties, for example, LMWH can exert anti-inflammatory effects by reducing the release of IL- $6 .{ }^{13-15,18}$

It was reported that IL-6 and IL-8 could cause hypercoagulation, leading to scattered fibrin clots, shortening the clot dissolution time and maximizing the dissolution rate. ${ }^{19}$ It was also observed that severe COVID-19 patients had higher levels of IL- $6,{ }^{7}$ suggesting that the hypercoagulation status of COVID-19 patients may be related to the elevated levels of cytokines. In previous studies of patients with COVID-19, D-dimer levels were significantly elevated in patients admitted to the ICU with severe disease. ${ }^{20}$ Higher levels of D-dimer and FDP in fatal cases have been reported, ${ }^{21}$ and there was certain correlation between D-dimer and COVID-19 severity.22 However, there is currently no conclusive evidence supporting the use of D-dimer as an evaluation index. ${ }^{23-25} \mathrm{~A}$ broad sample analysis is required 
to determine whether D-dimer is associated with COVID-19 severity. Therefore, the present study does not consider this parameter as an evaluation index for disease progression. The average values of D-dimer and FDP before treatment was higher in the LMWH group than in the control group (3.75, $1 \cdot 23, p<0 \cdot 01 ; 14 \cdot 35,4 \cdot 05, p<0 \cdot 01)$, therefore LMWH was applied. Because this trial is a retrospective analysis, we did not intervene in the type of treatment given to the patients, inferring that the purpose of medication in the LMWH group was to improve hypercoagulability. Because D-dimer and FDP are not considered as factors that designate patient's disease progression, their levels had no effect on subsequent analysis of the results.

Apart of its anticoagulant activity, there are other routes to explain a favorable effect of LMWH on COVID-19 patients. Heparan sulfate (HS), a linear polyanionic polysaccharide, is a major constituent of all mammalian cells and tissues. ${ }^{26}$ It highly resembles heparin and LMWH in its structural properties and sugar composition. ${ }^{26}$ Importantly, HS has been known to serve as the first point of contact between target cells and a large number of human viruses (i.e., dengue virus, hepatitis $\mathrm{C}$ virus, human immunodeficiency virus, human papilloma virus, herpes viruses), ${ }^{27,28}$ including the SARS-CoV-2 virus. ${ }^{29,30} \mathrm{~A}$ very recent online paper has used surface plasmon resonance and circular dichroism and showed that the SARS-CoV-2 Spike S1 protein receptor binding domain interacts with heparin. ${ }^{30}$ Heparin, LMWH and heparin-like compounds have been shown to efficiently compete with HS and thereby attenuate viral attachment and infection, ${ }^{31}$ providing a straightforward explanation for the anti-viral effect of LMWH in clinical settings.

Importantly, as clarified below, recent studies expanded the established role of HS from a viral attachment molecule to an essential receptor required for entry. Heparin, LMWH and non-anticoagulants species of heparin are known to inhibit the enzymatic activity of heparanase, ${ }^{32}$ the sole HS-degrading endoglycosidase, shown recently to promote viral infection and spread. ${ }^{33-35}$ It appears that heparanase behaves as a molecular switch in viral infection, which transforms the cell from a virus-permissive mode in which viral attachment and entry are favored, to a virus-deterring mode which allows for viral detachment and egress from cells. ${ }^{33}$ Briefly, it was found that upregulation and activation of heparanase is a strategy common to a broad range of viral species (i.e., PRRSV, vaccinia virus) to increase egress, spread and transmission. ${ }^{35}$ Interestingly, it appears that heparanase plays a role also in driving the undesirable cytokine storm discussed above. In individuals with SARS-CoV-2 infection, the level of inflammatory cytokines is markedly higher than normal and held responsible for the severity of the disease. Agelidis et al. documented that upon HSV-1 infection, heparanase translocate to the nucleus of the infected cells and promotes inflammatory signaling, mediated primarily via NF- $\mathrm{\kappa B} .{ }^{35}$ In fact, transcription of IL-6 was significantly decreased after treatment with an inhibitor of heparanase enzymatic activity. ${ }^{35}$ LMWH which inhibits heparanase activity $^{32}$ may have a similar effect, possibly providing a mechanistic explanation for the decrease in IL-6 that we observed in the LMWH treated patients. Collectively, the above considerations suggest that heparanase inhibitors (i.e., LMWH) may be an effective strategy in a therapeutic or combination therapy against viral infection, including COVID-19. Additional studies showed that inhibition of the glycocalyx-degrading enzymes sialidase, cathepsin $\mathrm{L}$ and heparanase, using a combination therapy of zanamavir, cathepsin-L and heparanase inhibitors, decreased vascular leakage after exposure to the influenza virus NS1 protein in vitro and in vivo. ${ }^{36}$ It will be interesting to see if an analogous therapeutic inhibition of glycocalyx breakdown can provide similar benefit clinically.

Several studies have recommended CRP and lymphocyte\% (LYM\%) as indices for evaluating the effectiveness of clinical drugs or treatments. ${ }^{5,37,38}$ In the various analyses applied in this study, there 
was no statistically significant difference in CRP levels between the groups, indicating that LMWH treatment has no effect on this parameter. Notably, LYM\% was higher in the LMWH group compared to the Control group ( $p<0 \cdot 001)$, consistent with the results of Derhaschnig et al. ${ }^{39}$ This suggests that LMWH can increase LYM\% in patients with COVID-19 and thereby improve their condition. Furthermore, it was reported that proinflammatory cytokines, such as TNF $\alpha$ and IL-6, can induce lymphopenia. ${ }^{6}$ Hence, the decrease in IL-6 (Fig. 4B) may contribute to the increase in LYM\% observed in the LMWH treated patients.

IL-6 levels in severely ill patients with COVID-19 are significantly higher than in patients with mild disease. ${ }^{7}$ Transition from mild to severe conditions in patients with COVID-19 occur when cytokine levels reach and/or exceed a certain threshold, leading to a cytokine release syndrome. ${ }^{8}$ Hence, reducing IL-6 release is expected to attenuate the cytokine storm syndrome caused by the virus, ${ }^{9}$ thereby improving the condition of patients with COVID-19. LMWH was reported to reduce the release of IL- 6 in the body by inhibiting the expression of nuclear factor $\kappa \mathrm{B}(\mathrm{NF}-\kappa \mathrm{B}) \cdot{ }^{13-15}$ Measuring the levels of proinflammatory cytokines in COVID-19 patients, we have found a marked decrease in the levels of IL- 6 in the LMWH treated patients compared to the patients without LMWH treatment $(p<0 \cdot 001)$, consistent with the proposed protective effect of LMWH. Changes in other inflammatory factors were not statistically significant. In addition, IL-6 can bind to HS on the cell surface, yielding a sufficiently high local concentration to activate signaling receptors, ${ }^{40}$ protect them against proteolysis, and promote paracrine action. ${ }^{18,41}$ An earlier study has reported that heparin binds IL-6, with affinity much higher than that of $\mathrm{HS},{ }^{18}$ thereby reducing its availability to its receptor complex. It, therefore, appears that LMWH reduces both the release of IL- 6 and its biological activity.

Collectively, LMWH not only improves the coagulation dysfunction of COVID-19 patients, ${ }^{42}$ but exerts an anti-inflammatory effect by means of reducing IL-6 and increasing LYM\%. We, therefore, favor the use of LMWH as a potential therapeutic drug for the treatment of COVID-19. We also suggest that non-anticoagulant species of LMWH that can be applied at high doses should be considered as a complement to conventional LMWH. To further support this conclusion, we are conducting a prospective clinical study to evaluate the efficacy and safety of one LMWH (enoxaparin) in the treatment of hospitalized adult patients with COVID-19 (Chinese Clinical Trial Registry, number:chiCTR2000030700), with the objective of providing a more powerful reference for the treatment conditions.

Limitations: This study has several limitations. First, due to the retrospective design, we were unable to control the time intervals between examinations of the various indices in patients and the LMWH treatment schedule. Likewise, we could not estimate and manage the effective dose and timing of LMWH. Second, there were no critical cases in the two groups of patients; the treatment outcome of all cases was improvement and discharge, and there were no deaths. Finally, the findings are limited by the sample size and single-center design of our study.

Contributors: CS, JPL and YZ conceptualized and designed the study, and CS and YZ had full access to all data, and took responsibility for data integrity and accuracy of the analysis. CS, CW, HX, JPL and IV wrote the manuscript. CY, FC and FZ reviewed the manuscript. FC, YH, TT and BD performed the statistical analysis. All authors contributed to data acquisition, analysis and interpretation, and approved the final version for submission.

Declaration of Interest: All authors declare no competing interests. 
Acknowledgements: This work was supported by the National Natural Science Foundation of China (No. 81603037 to SC) and the National Key Research and Development Plan of China(2017YFC0909900).

Patient consent for publication: Not required

Ethics approval The human study was approved the Research Ethics Committee of Union Hospital, Tongji Medical College, Huazhong University of Science and Technology.

\section{References}

1. Wang C, Horby PW, Hayden FG, et al. A novel coronavirus outbreak of global health concern. Lancet. 2020, 395(10223):470-473.

2. Huang CL, Wang YM, Li XW, et al. Clinical features of patients infected with 2019 novel coronavirus in Wuhan, China. Lancet. 2020, 395(10223):497-506.

3. De Wit E, van Doremalen N, Falzarano D, Munster VJ. SARS and MERS: recent insights into emerging coronaviruses. Nat Rev Microbiol. 2016;14(8):523-534.

4. Zhou W, Liu YS, Tian DD, et al. Potential benefits of precise corticosteroids therapy for severe 2019-nCoV pneumonia. Signal Transduction and Targeted Therapy. 2020;5:18.

5. Li Tan, Qi Wang, Duanyang Zhang, et al. Lymphopenia predicts diseaseseverity of COVID-19:a descriptive and predictive study. MedRxiv.2020.03.01.20029074.

6. Liao YC, Liang WG, Chen FW, Hsu JH, Yang JJ, Chang MS. IL-19 induces production of IL-6 and TNF-alpha and results in cell apoptosis through TNF-alpha. J Immunol. 2002;169(8): 4288-97.

7. Wan SX, Yi QJ, Fan SB, et al. Characteristics of lymphocyte subsets and cytokines in peripheral blood of 123 hospitalized patients with 2019 novel coronavirus pneumonia (NCP). MedRxiv. 2020[2020-02-24].

8. Shimabukuro-Vornhagen A, Gödel P, Subklewe M, et al. Cytokine release syndrome. J Immunother Cancer. 2018;6(1):56.

9. Tanaka T, Narazaki M, Kishimoto T. Immunotherapeutic implications of IL-6 blockade for cytokine storm. Immunotherapy. 2016;8(8):959-970.

10. Hunter CA, Jones SA. IL-6 as a keystone cytokine in health and disease. Nat Immunol. 2015;16:448-457.

11. Teachey DT, Lacey SF, Shaw PA, et al. Identification of Predictive Biomarkers for Cytokine Release Syndrome after Chimeric Antigen Receptor T-cell Therapy for Acute Lymphoblastic Leukemia. Cancer Discov. 2016;6(6):664-679.

12. Wang QL, Shang XY, Zhang SL, et al. Effects of inhaled low molecular weight heparin on airway allergic inflammation in aerosol-ovalbumin-sensitized guinea pigs. Jpn J Pharmacol. 2000;82(4):326-330.

13. Qian Y, Xie H, Tian R, Yu K, Wang R. Efficacy of low molecular weight heparin in patients with acute exacerbation of chronic obstructive pulmonary disease receiving ventilatory support. COPD. 2014;11(2):171-176.

14. Yina Liu, Shengtian $\mathrm{Mu}, \mathrm{Xu} \mathrm{Li}$, Yingjian Liang, Liang Wang, Xiaochun Ma. Unfractionated Heparin Alleviates Sepsis-Induced Acute Lung Injury by Protecting Tight Junctions. J Surg Res. 2019;6(238): 175-185.

15. Li X, Ma Y, Chen T, Tang J, Ma X. Unfractionated heparin inhibits lipopolysaccharide-induced expression of chemokines in human endothelial cells through nuclear factor- $\mathrm{KB}$ signaling pathway. 
Zhonghua Wei Zhong Bing Ji Jiu Yi Xue. 2016;28(2):117-121.

16. Dongmei Rao. Research progress on cytokine storm induced by pathogen infection. Medical Information. 2014;27(2):480-481.

17. Qian Chen, Yao Wang, Fangzhou Jiao, et al. Discussion on the mechanism and intervention strategy of inflammatory storm of new coronavirus pneumonia. Chinese Journal of Infectious Diseases. 2020;38(2020-02-26).

18. Mummery RS, Rider CC. Characterization of the heparin-binding properties of IL-6. J Immunol. 2000;165(10):5671-5679.

19. Bester J, Matshailwe C, Pretorius E. Simultaneous presence of hypercoagulation and increased clot lysis time due to IL-1 $\beta$, IL-6 and IL-8. Cytokine. 2018;110:237-242.

20. Huang C, Wang Y, Li X, et al. Clinical features of patients infected with 2019 novel coronavirus in Wuhan, China. Lancet. 2020;395(10223):497-506.

21. Tang N, Li D, Wang X, Sun Z. Abnormal coagulation parameters are associated with poor prognosis in patients with novel coronavirus pneumonia [published online ahead of print, 2020 Feb 19]. J Thromb Haemost. 2020;10.1111/jth.14768.

22. Li XY, Du B, Wang YS, et al. The keypoints in treatment of the critical coronavirus disease 2019 patient. Zhonghua Jie He He Hu Xi Za Zhi. 2020 Feb 29;43(0):E026.[Online ahead of print].

23. Querol-Ribelles JM, Tenias JM, Grau E, et al. Plasma d-dimer levels correlate with outcomes in patients with community-acquired pneumonia. Chest. 2004;126(4):1087-1092.

24. Snijders D, Schoorl M, Bartels PC,et al. D-dimer levels in assessing severity and clinical outcome in patients with community-acquired pneumonia. A secondary analysis of a randomised clinical trial. Eur J Intern Med. 2012;23(5):436-441.

25. Duarte JC, Tavares e Castro A, Silva R, et al. Prognostic value of plasma D-dimer level in adults with community-acquired pneumonia: a prospective study. Rev Port Pneumol (2006). 2015;21(4):218-219.

26. Esko JD, Lindahl U. Molecular diversity of heparan sulfate. J Clin Invest. 2001;108(2):169-173.

27. Chen Y, Maguire T, Hileman RE, et al. Dengue virus infectivity depends on envelope protein binding to target cell heparan sulfate. Nat Med. 1997;3(8):866-871.

28. Shukla D, Spear PG. Herpesviruses and heparan sulfate: an intimate relationship in aid of viral entry. J Clin Invest. 2001;108(4):503-510.

29. Milewska A, Nowak P, Owczarek K, et al. Entry of Human Coronavirus NL63 into the Cell. J Virol. 2018;92(3):e01933-17.

30. Mycroft-West, Dunhao Su, Stefano Elli, et al . The 2019 coronavirus (SARS-CoV-2) surface protein (Spike) S1 Receptor Binding Domain undergoes conformational change upon heparin binding. BioRxiv preprint.

31. Guo Y, Wang Z, Dong L, Wu J, Zhai S, Liu D. Ability of low-molecular-weight heparin to alleviate proteinuria by inhibiting respiratory syncytial virus infection. Nephrology (Carlton). 2008;13(7):545-553.

32. Vlodavsky I, Ilan N, Naggi A, Casu B. Heparanase: structure, biological functions, and inhibition by heparin-derived mimetics of heparan sulfate. Curr Pharm Des. 2007;13(20):2057-2073.

33. Hadigal SR, Agelidis AM, Karasneh GA, et al. Heparanase is a host enzyme required for herpes simplex virus-1 release from cells. Nat Commun. 2015;6:6985.

34. Khanna M, Ranasinghe C, Browne AM, Li JP, Vlodavsky I, Parish CR. Is host heparanase required for the rapid spread of heparan sulfate binding viruses?. Virology. 2019;529:1-6. 
medRxiv preprint doi: https://doi.org/10.1101/2020.03.28.20046144; this version posted April 15, 2020. The copyright holder for this preprint

(which was not certified by peer review) is the author/funder, who has granted medRxiv a license to display the preprint in perpetuity.

It is made available under a CC-BY-NC-ND 4.0 International license.

35. Agelidis AM, Hadigal SR, Jaishankar D, Shukla D. Viral Activation of Heparanase Drives Pathogenesis of Herpes Simplex Virus-1. Cell Rep. 2017;20(2):439-450.

36. Glasner DR, Ratnasiri K, Puerta-Guardo H, Espinosa DA, Beatty PR, Harris E. Dengue virus NS1 cytokine-independent vascular leak is dependent on endothelial glycocalyx components. PLoS Pathog. 2017;13(11):e1006673.

37. Fei Zhou, Ting Yu, Ronghui Du, et al. Clinical course and risk factors for mortality of adult inpatients with COVID-19 in Wuhan, China: a retrospective cohort study. Lancet. 2020 Mar 12:S0140-6736(20)30606-1. Online ahead of print.

38. Xun Li, Luwen Wang, Shaonan Yan.et al. Clinical characteristics of 25 death cases infected with COVID-19 pneumonia: a retrospective review of medical records in a single medical center, Wuhan, China. MedRxiv. 2020.02.25.

39. Derhaschnig U, Pernerstorfer T, Knechtelsdorfer M, Hollenstein U, Panzer S, Jilma B. Evaluation of antiinflammatory and antiadhesive effects of heparins in human endotoxemia. Crit Care Med. 2003;31(4):1108-1112.

40. Bernfield M, Götte M, Park PW, et al. Functions of cell surface heparan sulfate proteoglycans. Annu Rev Biochem. 1999;68:729-777.

41. Sarrazin S, Lamanna WC, Esko JD. Heparan sulfate proteoglycans. Cold Spring Harb Perspect Biol. 2011;3(7):a004952.

42. Tang N, Bai H, Chen X, Gong J, Li D, Sun Z. Anticoagulant treatment is associated with decreased mortality in severe coronavirus disease 2019 patients with coagulopathy [published online ahead of print, 2020 Mar 27]. J Thromb Haemost. 2020;10.1111/jth.14817. 


\section{Figure captions}

Figure 1. Possible mechanism of anti-inflammatory effects of LMWH in patients with COVID-19. Under conventional antiviral treatment regimens, LMWH improves hypercoagulability, inhibits IL-6 release, and attenuates IL-6 biological activity. It has potential antiviral effects and helps delay or block inflammatory cytokine storms. LMWH can increases the LYM\% in the patients. The multiple effects of LMWH encourages its application for the treatment of COVID-19 patients.

Figure 2. Flowchart of inclusion and exclusion criteria for patients with COVID-19.

Based on strict inclusion and exclusion criteria, 42 patients with COVID-19 treated at the hospital between February 1 and March 15, 2020, were selected for the study, of which 21 underwent LMWH treatment (LMWH group) and 21 did not (Control group) during hospitalization.

Table 1. LMWH use in treating conditions of the 21 patients with COVID-19.

Details of the dose, frequency, route of administration, and days of use of LMWH in the LMWH group.

Table 2. General characteristics of all the enrolled patients with COVID-19.

There were no significant differences in age, sex, comorbidities, onset symptoms, time from hospitalization to virus shedding, length of hospital stay, antiviral treatment, and disease progression between the two groups. Data are median (IQR) or $n(\%)$. p values are for comparing the LMWH group and Control group. NA = not applicable.

Figure 3. Effect of LMWH on complete blood count, coagulation profile, and CRP in the enrolled patients with COVID-19.

A-N: Red blood cells (A), white blood cells (B), monocytes\% (C), neutrophils\% (D), lymphocytes\% (E), TT (F), APTT (G), PT (H), D-dimer (I), FDP (J), FIB (K), AT III (L), INR (M) and CRP (N) levels in patients with COVID-19. Data are expressed as mean \pm standard deviation (SD) $(\mathrm{n}=21) . \mathrm{C} 1$ vs. H1 or $\mathrm{C} 2$ vs. H2, ${ }^{\mathrm{a}} p<0 \cdot 05,{ }^{\text {aa }} p<0 \cdot 01$, ${ }^{\text {aaa }} p<0.001$; $\mathrm{C} 1$ vs. $\mathrm{C} 2$ or $\mathrm{H} 1$ vs. $\mathrm{H} 2,{ }^{\mathrm{b}} p<0 \cdot 05,{ }^{\mathrm{bb}} p<0 \cdot 01,{ }^{\mathrm{bbb}} p$ $<0.001$; C3 vs. H3, ${ }^{\mathrm{c}} p<0.05,{ }^{\mathrm{cc}} p<0.01,{ }^{\mathrm{ccc}} p<0.001$. (C1: Control group, indices at admission; $\mathrm{C} 2$ : Control group, indices at discharge; $\mathrm{C} 3$ : Control group, changes in indices during hospitalization; $\mathrm{H} 1$ : LMWH group, indices before LMWH treatment; H2: LMWH group, indices after LMWH treatment; H3: LMWH group, changes in indices before and after LMWH treatment.).

Figure 4. Effect of LMWH on inflammatory cytokines in the enrolled patients with COVID-19.

A-F: IL-2 (A), IL-6 (B), TNF- $\alpha$ (C), IL-4 (D), IL-10 (E), and IFN- $\gamma$ ( F) levels in the two groups of patients with COVID-19. Data are expressed as mean \pm standard deviation (SD) $(\mathrm{n}=21)$. C1 vs. H1 or $\mathrm{C} 2$ vs. H2, a $p<0.05$, ${ }^{\text {aa }} p<0 \cdot 01$, aaa $p<0.001$; C1 vs. $\mathrm{C} 2$ or H1 vs. $\mathrm{H} 2,{ }^{\mathrm{b}} p<0.05,{ }^{\mathrm{bb}} p<0 \cdot 01,{ }^{\mathrm{bbb}} p<$ $0 \cdot 001$; $\mathrm{C} 3$ vs. $\mathrm{H} 3,{ }^{\mathrm{c}} p<0 \cdot 05,{ }^{\mathrm{cc}} p<0 \cdot 01,{ }^{\mathrm{ccc}} p<0 \cdot 001$. (C1: Control group, indices at admission; $\mathrm{C} 2$ : Control group, indices at discharge; $\mathrm{C} 3$ : Control group, changes in indices during hospitalization; $\mathrm{H} 1$ : LMWH group, indices before LMWH treatment; H2: LMWH group, indices after LMWH treatment; H3: LMWH group, changes in indices before and after LMWH treatment). 
SARS-CoV-2 entry

ACE2 receptor
Competing with HSPG for the binding of SARS-COV-2 and preventing virus invasion

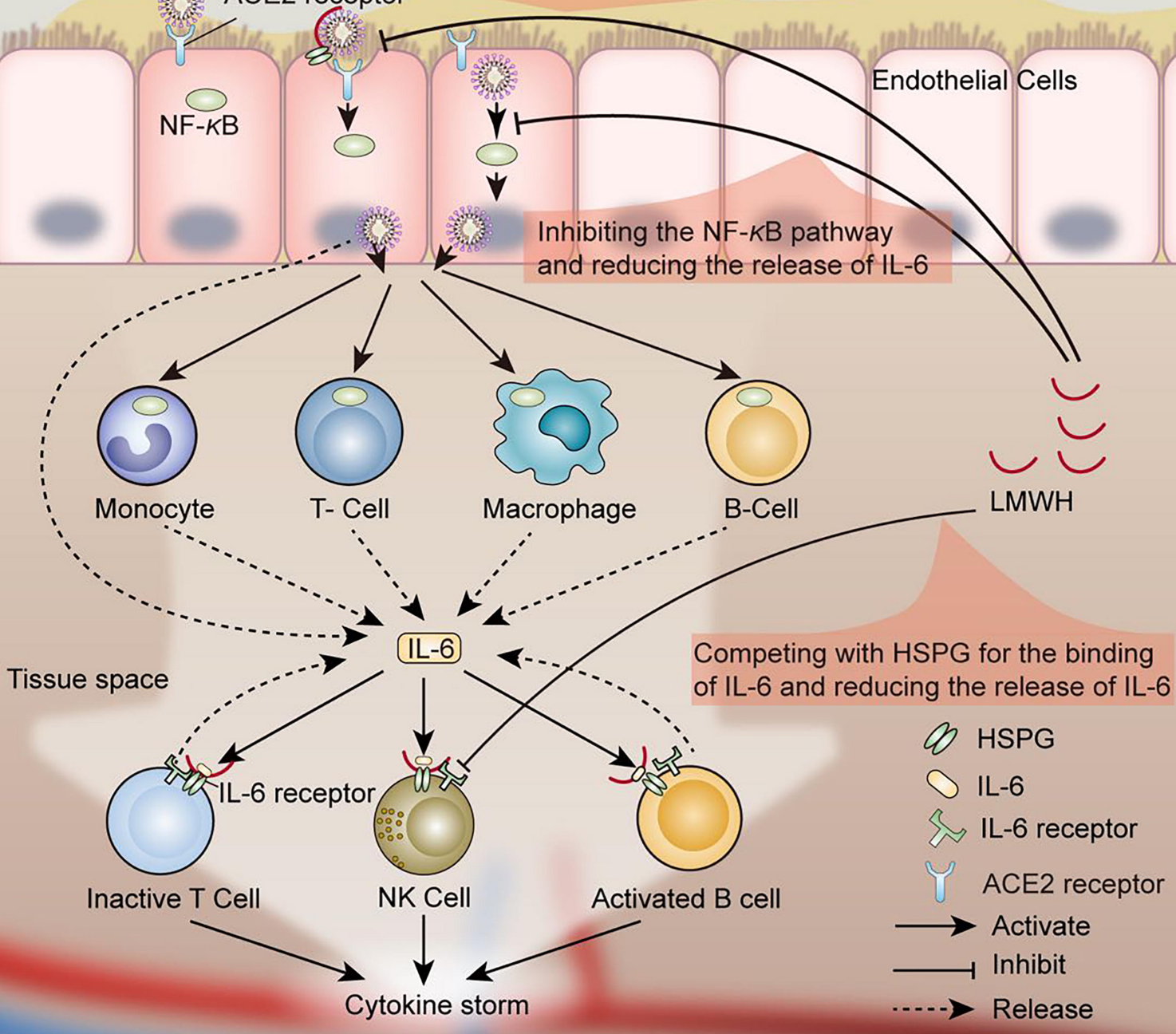




\section{Hospitalized patients}

Inclusion criteria:

(1) Patients were diagnosed as COVID-19 according to the New Coronavirus Pneumonia Diagnosis Program (7th edition) published by the National Health Commission of China;

(2)The clinical classification was severe, including any of the following: shortness of breath, RR $\geq 30 \mathrm{bpm}$, blood oxygen saturation $\leq 93 \%$ (at rest), $\mathrm{PaO} 2 / \mathrm{FiO} 2 \leq 300 \mathrm{mmHg}$, or pulmonary inflammation that progresses significantly within 24 to 48 hours $>50 \%$;

(3)Age $\geq 18$ years old;

(4) Patients with no previous respiratory diseases such as bronchiectasis and bronchial asthma;

(5) Patients who had not received immunosuppressive agents and glucocorticoid therapy during hospitalization.

\section{Exclusion criteria:}

(1) Patients with severe systemic diseases and other acute/chronic infectious diseases;

(2) Patients with liver/kidney dysfunction and congenital heart disease;

(3) Patients who received LMWH treatment within the last three months;

(4)Patients with a previous history of mental illness;

(5) Pregnant or lactating women;

(6) Critical patients or patients requiring ICU care;

(7) Patients with allergy to LMWH or contraindication to LMWH.

\section{$\downarrow$}

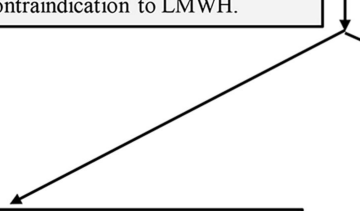

Patients who received LMWH treatment during hospitalization (LMWH Group, $\mathbf{n = 2 1}$ ) 
$\mathrm{H} 1$

$\mathrm{H} 2$

$\mathrm{H} 3$

$\mathrm{H} 4$

H5

H6

$\mathrm{H} 7$

$\mathrm{H} 8$

H9

$\mathrm{H} 10$

$\mathrm{H} 11$

$\mathrm{H} 12$

$\mathrm{H} 13$

$\mathrm{H} 14$

H15

$\mathrm{H} 16$

$\mathrm{H} 17$

$\mathrm{H} 18$

H19

$\mathrm{H} 20$

$\mathrm{H} 21$
Enoxaparin sodium injection 4000AxaIU qd $\mathrm{i} \cdot \mathrm{h} \cdot$

Enoxaparin sodium injection 4000AxaIU qd $\mathrm{i} \cdot \mathrm{h} \cdot$

Enoxaparin sodium injection 4000AxaIU qd $\mathrm{i} \cdot \mathrm{h} \cdot$

Enoxaparin sodium injection 4000AxaIU qd $\mathrm{i} \cdot \mathrm{h}$.

Enoxaparin sodium injection 4000AxaIU qd $\mathrm{i} \cdot \mathrm{h}$.

Nadroparin calcium injection 4100AxaIU qd $\mathrm{i} \cdot \mathrm{h} \cdot$

Enoxaparin sodium injection 4000AxaIU qd $\mathrm{i} \cdot \mathrm{h}$.

LMWH sodium injection 5000IU once $\mathrm{i} \cdot \mathrm{h}$.

Enoxaparin sodium injection 4000AxaIU qd $\mathrm{i} \cdot \mathrm{h}$.

Enoxaparin sodium injection 4000AxaIU qd $\mathrm{i} \cdot \mathrm{h}$.

Enoxaparin sodium injection 4000AxaIU qd $\mathrm{i} \cdot \mathrm{h}$.

Enoxaparin sodium injection 2000AxaIU qd $\mathrm{i} \cdot \mathrm{h}$.

Enoxaparin sodium injection 2000AxaIU qd $\mathrm{i} \cdot \mathrm{h}$.

Nadroparin calcium injection 4100AxaIU qd $\mathrm{i} \cdot \mathrm{h}$.

Nadroparin calcium injection 4100AxaIU qd $i \cdot h \cdot$

Enoxaparin sodium injection 4000AxaIU qd $\mathrm{i} \cdot \mathrm{h} \cdot$

Nadroparin calcium injection $4100 \mathrm{AxaIU}$ qd $\mathrm{i} \cdot \mathrm{h} \cdot$

LMWH sodium injection 5000IU qd $\mathrm{i} \cdot \mathrm{h}$.

Enoxaparin sodium injection $4000 \mathrm{AxaIU} \mathrm{qd} \mathrm{i} \cdot \mathrm{h} \cdot$

Enoxaparin sodium injection 4000AxaIU qd $\mathrm{i} \cdot \mathrm{h}$.

Enoxaparin sodium injection 4000AxaIU qd $\mathrm{i} \cdot \mathrm{h}$.
10

10

14

13

17

9

2

1

16

19

14

19

22

11

13

8

19

8

8

7

10 


\section{Characteristics}

Age, years

Sex

Female

Male

Comorbidity

Hypertension

Diabetes

Cardiovascular disease

Chronic obstructive lung disease

Carcinoma

Chronic kidney disease

Other

\section{Signs and symptoms}

Fever (temperature $\geqslant 37.3^{\circ} \mathrm{C}$ )

Cough

Sputum

Chest distress or asthma

Myalgia

Fatigue

Anorexia

Diarrhoea

Nausea or vomiting

Respiratory rate $\geqslant 30$ breaths per min

Pulse $\geqslant 125$ beats per min

Systolic blood pressure $<90 \mathrm{mmHg}$

\section{Antiviral therapy}

Arbidol

Recombinant Human Interferon $\alpha 2 \mathrm{~B}$

(aerosol inhalation)

Ribavirin

Lopinavir/Ritonavir

Traditional Chinese medicine decoction

\section{Disease progression}

Improved

Invariable

Deteriorative

Time from hospitalization to virus shedding

$15(71 \%)$

$13(62 \%)$

$0 \cdot 51$

$9(43 \%)$

$6(29 \%)$

$11(52 \%)$

$2(10 \%)$

$8(38 \%)$

$6(29 \%)$

$2(10 \%)$

$2(10 \%)$

0

0

0

$18(86 \%)$

$20(95 \%)$

$0 \cdot 29$

$6(29 \%)$

$6(29 \%)$

$1 \cdot 00$

$2(10 \%)$

0

$0 \cdot 15$

$2(10 \%)$

0

$0 \cdot 15$

$11(52 \%)$

$9(43 \%)$

$0 \cdot 54$

$\begin{array}{ll}21(100 \%) & 21 \\ 0 & 0\end{array}$

NA

$0 \quad \mathrm{NA}$

$20 \cdot 0(11 \cdot 0-31 \cdot 0) \quad 19 \cdot 0(12 \cdot 0-30 \cdot 0) \quad 0 \cdot 46$

- NA
after the onset of the COVID-19, days 

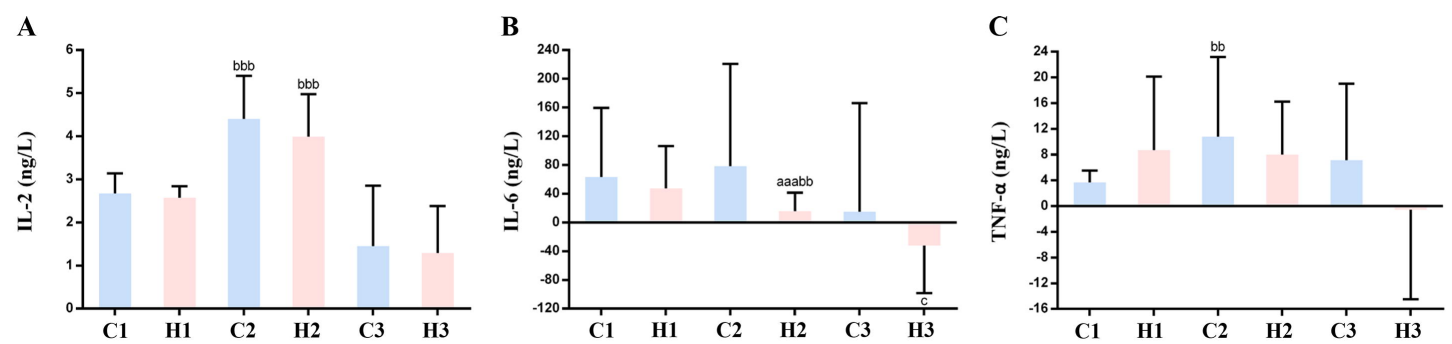

D

E

F
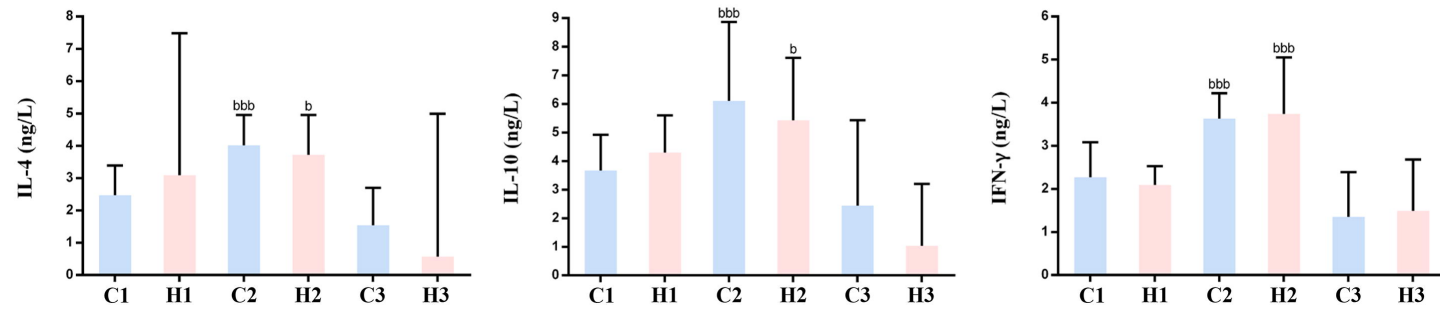